\title{
Toolbox-based routines for Macintosh timing and display
}

\author{
RONALD A. RENSINK \\ University of British Columbia, Vancouver, British Columbia, Canada
}

(Student Award Winner for 1989)

\begin{abstract}
Pascal routines are described for several real-time operations on Macintosh computers. Methods are presented for millisecond timing and for high-speed transfer of arbitrary bit-image displays to the screen. All routines are based on Toolbox procedures available on virtually all Macintosh computers.
\end{abstract}

Macintosh computers allow a wide variety of fast and flexible graphics operations to be carried out at relatively low cost. Several easy-to-use graphics editors, such as FullPaint and PixelPaint, allow complex images to be produced on the Mac with relatively little effort. Consequently, the Mac has been used extensively for applications involving sophisticated visual displays.

There is one problem, however. The Toolbox instructions for high-level control of the Mac do not allow many real-time operations to be easily programmed. Furthermore, much of the documentation on the relevant Toolbox procedures is obscure in key places, and in some cases, important points are completely omitted. The programming of operations such as millisecond timing and fast display has therefore relied on a combination of special assembly-language routines (see, e.g., Lane \& Ashby, 1987; Westall, Perkey, \& Chute, 1986) and special highlevel languages such as Rascal (Reed College, 1985). These approaches solve the problem, but at a high cost: they are usually designed for only one processor or machine architecture, and so are not always portable to other machines. Furthermore, the casual user must spend considerable time and energy to learn to use them correctly.

This article describes a different approach to the problem, one that is more in spirit with the philosophy of the Toolbox. It presents a description of how timing and display operations can be carried out and tested with available Toolbox routines. Pascal routines are given that show how the Time Manager can be used for millisecond

This work has been supported in part by the University of British Columbia's Centre for Integrated Computer Systems Research, and by the National Sciences and Engineering Research Council of Canada (via R. J. Woodham). The author would like to thank James T. Enns of the Department of Psychology for his encouragement and help, without which this paper could not have been written. Eric Ochs provided useful feedback on earlier drafts, and Jack Moxness supplied code that helped clarify the functioning of slots. Many thanks also to Bob Woodham of the Department of Computer Science for his support. Correspondence may be addressed to $\mathbf{R}$. A. Rensink, Laboratory for Computational Vision, Department of Computer Science, University of British Columbia, Vancouver, BC V6T IW5, Canada. timing. This allows input events to be timed with an accuracy limited only by the system hardware. Procedures are also given for the high-speed transfer of images from off-screen buffers onto the screen; for a Mac II with a $480 \times 640$ monitor, this allows an indefinite number of images to be displayed sequentially with each screen refresh. These techniques can be used as the basis of routines that will operate on all current models of the Macintosh computer, and, presumably, on all future models as well.

Not all timing and display applications can be foreseen in advance. Consequently, this article is somewhat tutorial. The code for each routine is presented along with the description of its operation, so that the reader may develop a feeling for how to use Toolbox routines for particular timing and display operations. To keep things simple, there is no error checking and only rudimentary input/output. All routines have been extensively tested and found to operate consistently on all Macintosh models currently supported by Apple-the Mac Plus, SE, II and IIx, and SE/30. Many are applicable to the $128 \mathrm{~K}$ and $512 \mathrm{~K}$ models as well, and all have been found to operate correctly on the enhanced version of the 512K. Placing these routines into the "shell" program TD__Tests (Figure 1) will allow them to be run and tested on any machine.

\section{MILLISECOND TIMING}

With the introduction of the Time Manager in the Mac Plus, Toolbox procedures became available to access the millisecond timer on the VIA chip. Previously, this was possible only with special assembly-language programs; for the $128 \mathrm{~K}$ and $512 \mathrm{~K}$ Macs, this is still the only way to do millisecond timing. These programs are described in great detail elsewhere (see, e.g., Westall et al., 1986), so they will not be discussed here. Rather, consideration will be limited to machines with a Time Manager on board-to all Macs currently supported by Apple, that is, as well as the enhanced version of the $512 \mathrm{~K}$.

The general characteristics of the Time Manager itself will not be described in great detail, unless they bear directly on issues of setting up and using millisecond timing. For other information about the Time Manager, see 
program TD_Tests;

uses \{Units depend on development system -- these are for Lightspeed[TH] Pascal \}

Memtypes, Quickdran, OSIntf, Toolint1, PackIntf, VideoInt1; const

MaxLong $=2147483647$;

TickFactor $=16.626822 ;$ ticks to $\mathrm{msec}\}$

NoSlot $=0 ;\{$ value used vhen no slot active

type

TMTaskPtr $=$-THTask;

VBLTaskPtr = -VBLTask;

var

Currevent : Eventrecord;

Errcode : integer; \{code returned for i/o errors \}

Delaystat : boolean; global llag for delay

TimerTask, TriggerTask, DelayTask : TMTask; \{timing tasks

TimerPtr, TriggerPtr, DelayPtr : TMTaskPtr; \{ ptrs to timing tasks \}

MainPort, ImagePort: grafPtr; $\{$ graphics ports $\}$

MainBuffer, ImageBuffer, BackBuffer: ptr; \{ ptrs to bit image buffers

MonitorX, Monitory : integer; \{dimensions of screen \}

CardWidth; \{ vidth of screen buffer (in bytes) \}

CurrMacType: integer: \{machine model \}

Smallscreen : boolean; \{ video system \}

Currslot : integer; \{slot identifier (large-screen Macs only) \}

SlotTask : SlotIntQElem; \{slot-interrupt task (large-screen Macs only)

SlotPtr : SQElemPtr; \{slot-task ptr (large-screen Macs only) \}

VideoTask : VBLTask; \{screen-counter task (large-screen Macs only) \}

Videoptr : VBLTaskPtr; \{ screen-counter ptr (large-screen Macs only) \}

videocount : longint; \{ global counter for screen refreshes \}

$\{\ldots$ setup and shutdown routines defined here ...

$\{\ldots$ timing and display routines defined here ... $\}$

begin $\{$ TD_Tests $\}$

$\{\ldots$ general initialization procedures placed here ... $\}$

setUpTimers; \{ set up Time Kanager tasks \}

setUpBuffers; \{ set up buffers containing images

setUpVCounter; \{ set up counter for screen refreshes

triggerTest; \{ test trigger-based timing \}

anchorTest; \{test anchor-based hybrid timing

delayTest; \{ test millisecond delay \}

inputTest; \{ test timing of keyboard input \}

screentest; $\{$ test fast display of image onto screen \}

shutDornvCounter;

shutDornBuffers;

shutDornTimers;

end; $\{$ TD_Tests $\}$

Figure 1. Shell program for timing and display procedures. 
pages IV-299-IV-301 of Inside Macintosh (Apple Computer, 1986).

\section{Basics}

The operation of the Time Manager is based on entries of the type TMTask that are placed in a special Time Manager queue. Each entry has a tmCount field, which contains the time (in msec) until the associated task is executed. This number is regularly decremented until it reaches zero, at which point the Time Manager executes the procedure pointed to by the tmAddr field of the task. It has been noted by Kieley and Higgins (1988) that tmCount is incorrectly specified as being of the type integer. It is actually a longint, and so can be used for du- rations as long as $2^{11}-1=2,147,483,647 \mathrm{msec}$, or roughly $596 \mathrm{~h}$.

It is worth mentioning here that the representation used in the tmCount field has a slight dependence on the version of operating system being used. For version 6.0.2 (and most earlier versions), the value in tmCount is exactly the time until the task is to be executed. However, in version 6.0.3 (and presumably later versions), the Time Manager uses a different representation, in which the time until execution is given by the value of the tmCount field divided by 49 . This is simple enough to compensate for, so that in what follows, only the procedures based on the direct representation will be given.

$\{h+\}\{$ asynchronous $\}$ procedure DummyProc; \{ dummy procedure for timers $\}$ begin

end;

$\{\mathbf{A}-\}$

$\{A+\}$ \{ anynchronous \} procedure DolayProc; \{ procodure for delay $\}$ begin

Delaystat := false; \{remove delay condition \} end;

$\{a-\}$

procedure setUpTimers;

var

tVal : longint;

begin $\{$ setUpTimers $\}$

TimerPtr : = OTimerTak;

TriggerPtr := OTriggerTask;

DelayPtr : = eDelayTask;

TimerTask.tmlddr := ODummyProc; \{dumy task \}

TriggerTask.tmAddr := ODummyProc; \{dumay task \}

DelayTask, tmaddr := 0.elayproc; \{change Delaystat shen activated \}

InsTime(QElemPtr(TimerPtr)); \{ install timing tasks into queue \}

InsTime (QEI OmPtr(TriggorPtr));

InsTime (QElemPtr (DelayPtr));

PrimeTime(QElemPtr(TimerPtr), MaxLong); \{ set tmCount fields \}

PrimeTime( $Q E$ elemPtr (TriggerPtr), 0 ):

PrimeTime(QElemPtr(DelayPtr), 0 );

tval : = tickCount +10 ;

repeat

until tickCount > tVal; \{ wait and reset TimerTask \}

PrimeTime(QElemPtr(TimerPtr), MaxLong);

end: \{ setUptimers $\}$

procedure shutDornTimers;

begin \{ shutDoontimers \}:

RuvTime(QE] emPtr (TimerPtr));

RmvTime (QElemptr(TriggerPtr));

RmvTime (QEl emPtr(DelayPtr));

end \{ shutDoonTimers \}; 
The procedure setUpTimers in Figure 2 shows how timing tasks can be set up. Here, TimerTask and TriggerTask are associated with an asynchronous null operation, while DelayTask invokes an asynchronous procedure that sets the global variable DelayStat to false. (Note that asynchronous tasks preserve the value of the A5 registers.) The pointers associated with these tasks are installed in the queue, and the tmCount fields are set by the PrimeTime procedure. Testing (on the basis of the tests described below) has shown that installing nonzero values of tmCount does not always cause activation of Time Manager updating. However, if the task is reinstalled after a delay of more than approximately 6 ticks, updating is carried out correctly; for caution, a delay of about 10 ticks is used in TD_Tests.

A routine for shutting down the timing tasks (shutDownTimers) is also given in Figure 2. It is important to call this procedure before exiting the program, because otherwise a system crash will occur.

\section{Timing}

Timing can be done by accessing the tmCount field of a TMTask. In TD_Tests, the global variable TimerTask is used exclusively for this purpose. The procedure milliCount (in Figure 3) shows how this value may be correctly accessed. Since the value of the tmCount field is always decremented by the Time Manager, subtraction of tmCount from MaxLong yields a positive value that constantly increases. Set up in this way, milliCount is analogous to tickCount.

The procedure triggerTest (Figure 3) is designed to test the consistency of millisecond timing with that based on ticks. Timing begins at the point where tickCount reaches the first mark, tVal1, and stops where it reaches the sec-

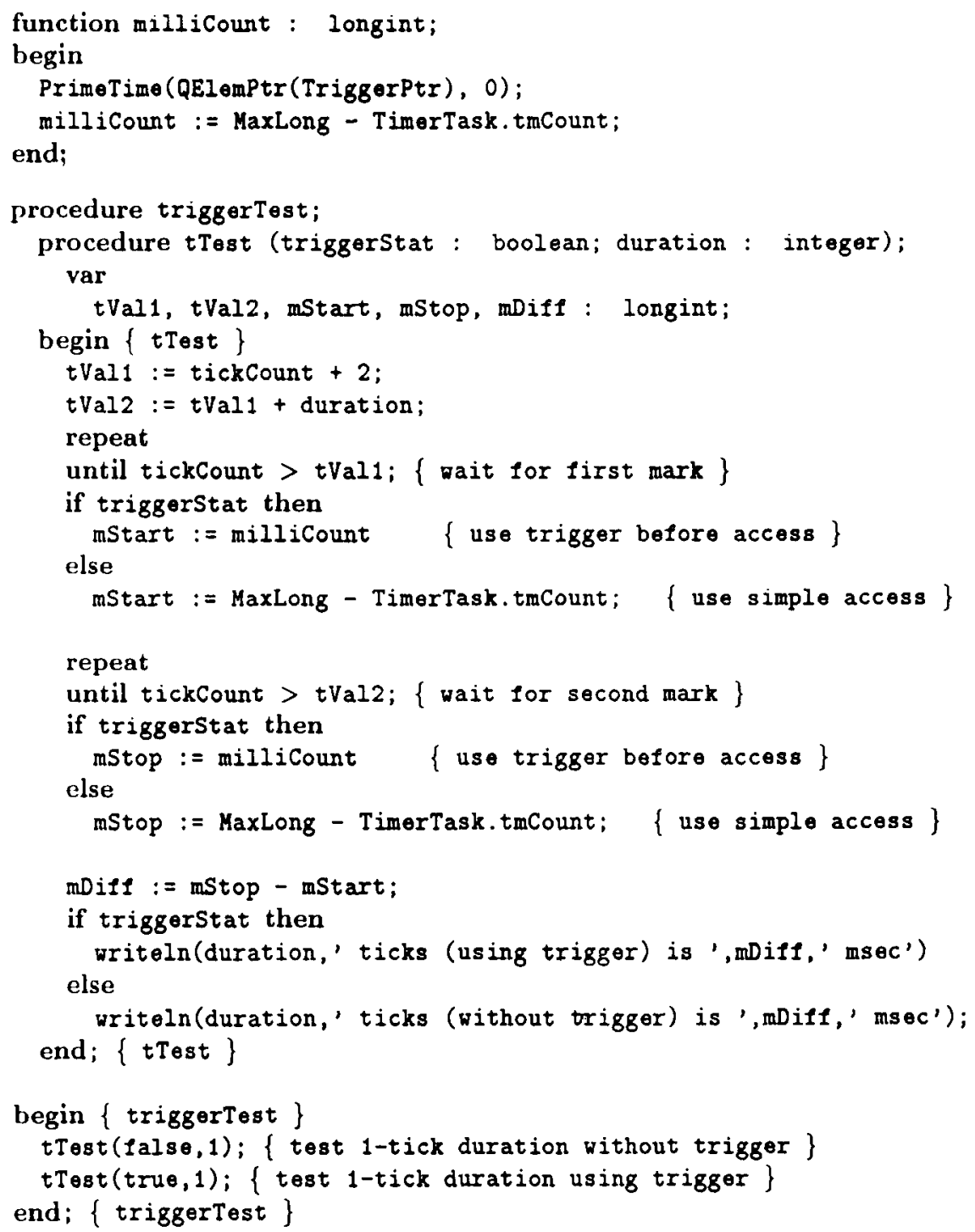

Figure 3. Procedure for triggered millisecond timing. 
ond mark, tVall + duration. Running triggerTest shows that a straightforward access of tmCount does not result in precise timing. Apparently (at least on the systems tested) tmCount fields are only updated every 5 ticks. Simple access of tmCount thus yields lower precision than tick timing does! To solve this problem, milliCount uses PrimeTime to install TriggerTask with a zero argument. This acts as a trigger, forcing the Time Manager to update the tmCount fields of all tasks in the queue to the nearest millisecond.
Testing based on triggerTest shows this method of timing to be accurate to within $1 \mathrm{msec}$ over intervals of about a second or so, depending on the particular machine. Beyond this range, errors of several milliseconds can accumulate. However, it is possible to avoid cumulative error via a hybrid timer that uses tick timing for coarse large-scale timing, together with simple millisecond timing for finer small-scale measurement.

The procedure anchorTest in Figure 4 provides an example of how this can be done. Coarse timing is done

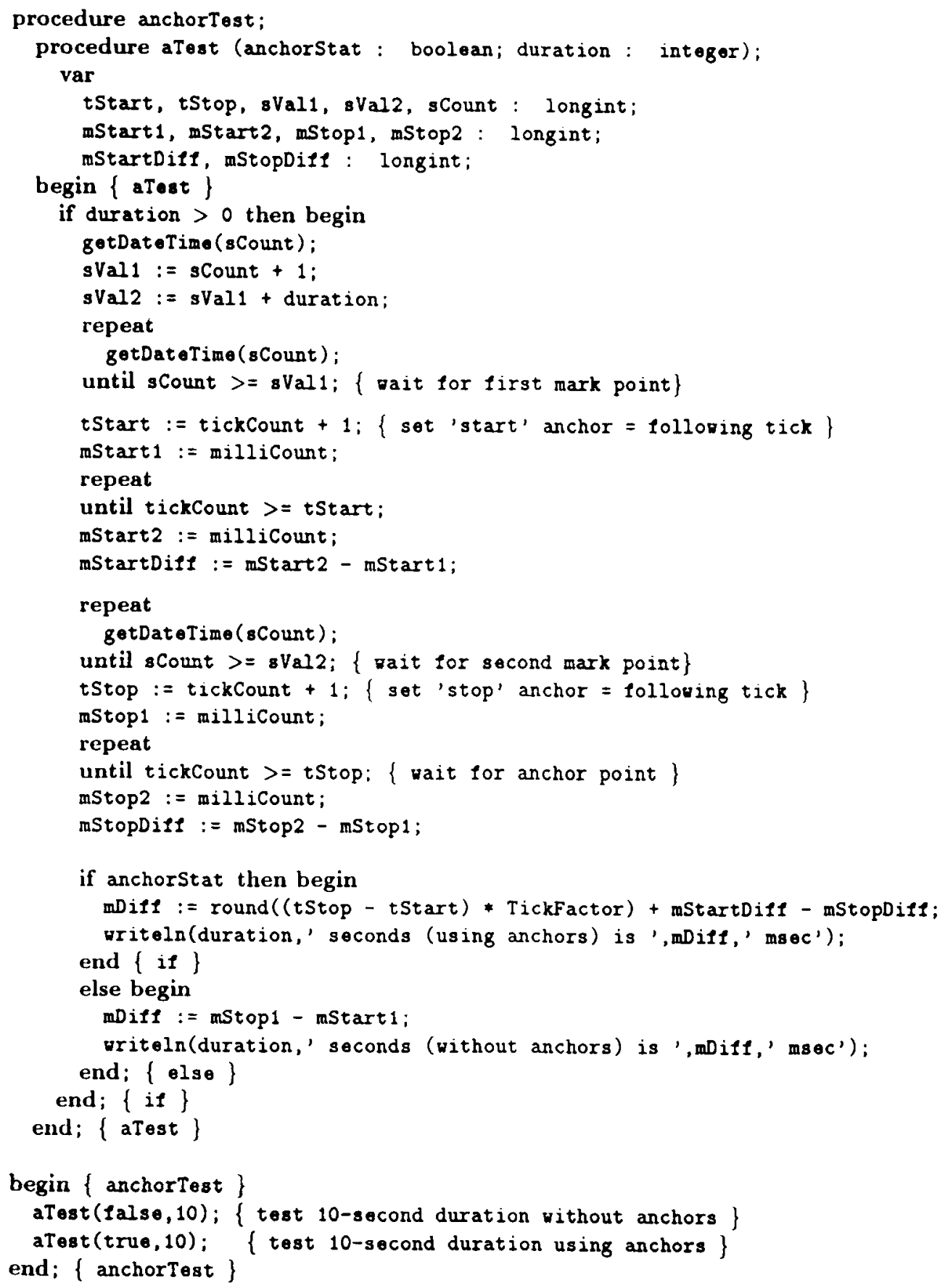


by taking the difference between anchor points, which are tickCount values near the actual starting and stopping times. The difference between the actual times and the anchors is then measured by simple millisecond timing; the upper limit to these differences must be less than a second or so, but otherwise there are no special constraints. The subroutine aTest in Figure 4 shows how the anchor points may be set. The first (tStart) is one more than the value of tickCount at the time the seconds counter (accessed by getDateTime) reaches the first mark, $\mathrm{sVal1}$; the difference $\mathrm{mStar} 2-\mathrm{mStart} 1$ consequently becomes the duration from the start of timing to the anchor point. A similar rationale is used to determine the point at which the seconds counter reaches the second mark, sVal2. The difference between the two mark points is then calculated by converting the difference between the anchors to milliseconds and adding or subtracting the appropriate small-scale measurements.

Testing with anchorTest shows that hybrid timing of internal operations can be carried out with millisecond accuracy over arbitrarily long stretches of time, regardless of the load (internal or external) placed upon the system.

This approach can also be used to time inputs, as is shown in the procedure inputTest (Figure 5). The event queue is first cleared. The procedure then loops until an event is detected by OSEventAvail; the loop is subsequently exited and the anchor point set to the value of tickCount. The millisecond timer is then triggered, and the difference between it and the time to the next tick is measured. Subtraction of this interval from $17 \mathrm{msec}$ (the time for a tick) yields the time from the anchor point.

Such timing is obviously at least as accurate as that based on ticks. Furthermore, repeated testing of inputTest shows the millisecond difference between anchor and detected response to be fairly evenly distributed between 0 and $16 \mathrm{msec}$. This is consistent with a very fast and uniform response by OSEventAvail to input events; if the system response to inputs has a standard deviation of a millisecond or so, such a distribution would be expected. Lane and Ashby (1987) report that for models earlier than the SE, the time from keypress to system interrupt takes a constant 6 msec. For the SE and Mac II, ADB control of keyboard input requires any keyboard event to be signaled within $260 \mu \mathrm{sec}$ of its occurrence. Provided that the keyboard is the active input device (i.e., the device that has last sent input to the system), and that no service requests are pending from any other device, the keyboard will be continuously polled-without using processor time-until a key is pressed (see pp. 11-9-11-16 and 11-

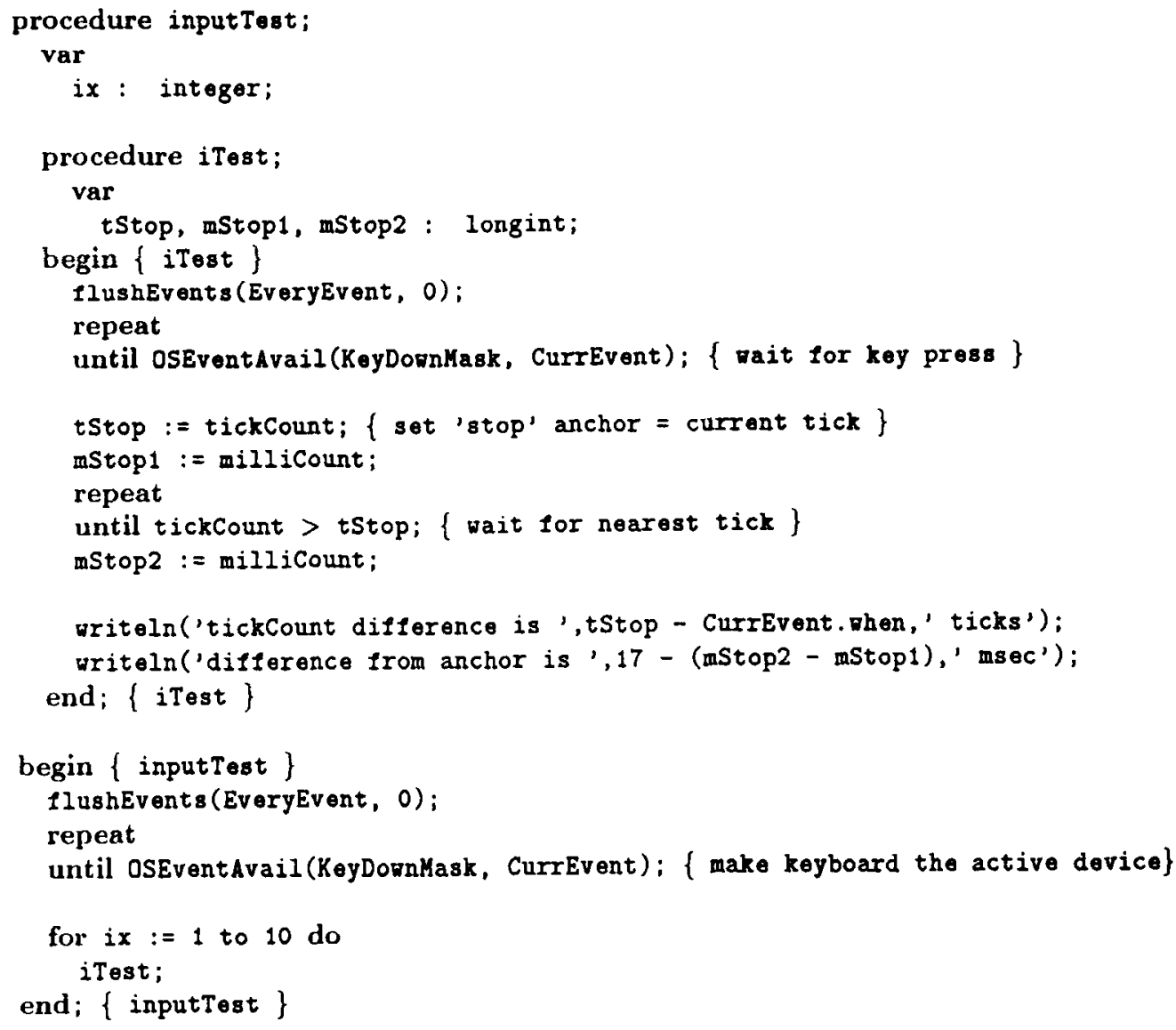


25 of the Macintosh Family Hardware Reference, Apple Computer, 1986). The documentation is somewhat vague on this point, but the polling rate will be at least once every $11 \mathrm{msec}$, and it may be much higher than that. Therefore, although proof cannot be given with the routines described here, it is plausible that high-accuracy timing of inputs can be achieved by this method. An exact measurement of this accuracy must await experiments based on external timers.

\section{Delays}

One other useful real-time capability is the control of delays to millisecond precision. The routine mDelay (Figure 6) shows how this can be done. Delay is carried out via DelayTask, which is devoted exclusively to this operation. This task calls the asynchronous procedure DelayProc, which sets the global flag DelayStat to false.

When mDelay is called, it places DelayTask in the Time Manager queue, and loops until DelayStat is set to false by DelayProc. Testing against the seconds timer (as done in delayTest) shows that delays of up to a second or so can be carried out to millisecond accuracy by this simple mechanism. Longer delays can be carried out using an anchor-based mechanism similar to that used for tıming.

\section{FAST DISPLAY}

For machines prior to the Mac II (viz., the $128 \mathrm{~K}, 512 \mathrm{~K}$. Mac Plus, and SE), displays can be changed at refresh rates by paging between the main and alternate video buffers. The method for doing this is presented on page III-20 of Inside Macintosh (Apple Computer, 1985). This method will not be described here any further than to point out that it is limited to two displays, and cannot be applied to Mac II-type machines. In this section, I will describe how these limitations can be overcome to allow sequential display of an indefinite number of bit images at refresh rates on all machines with standard display monitors.

It should be emphasized that this section applies only to bit (or binary) images, and not to full-color displays. Also, the way that bit images are created and represented will not be described in great detail. For more information about these images, see, for example, pages 1-142-

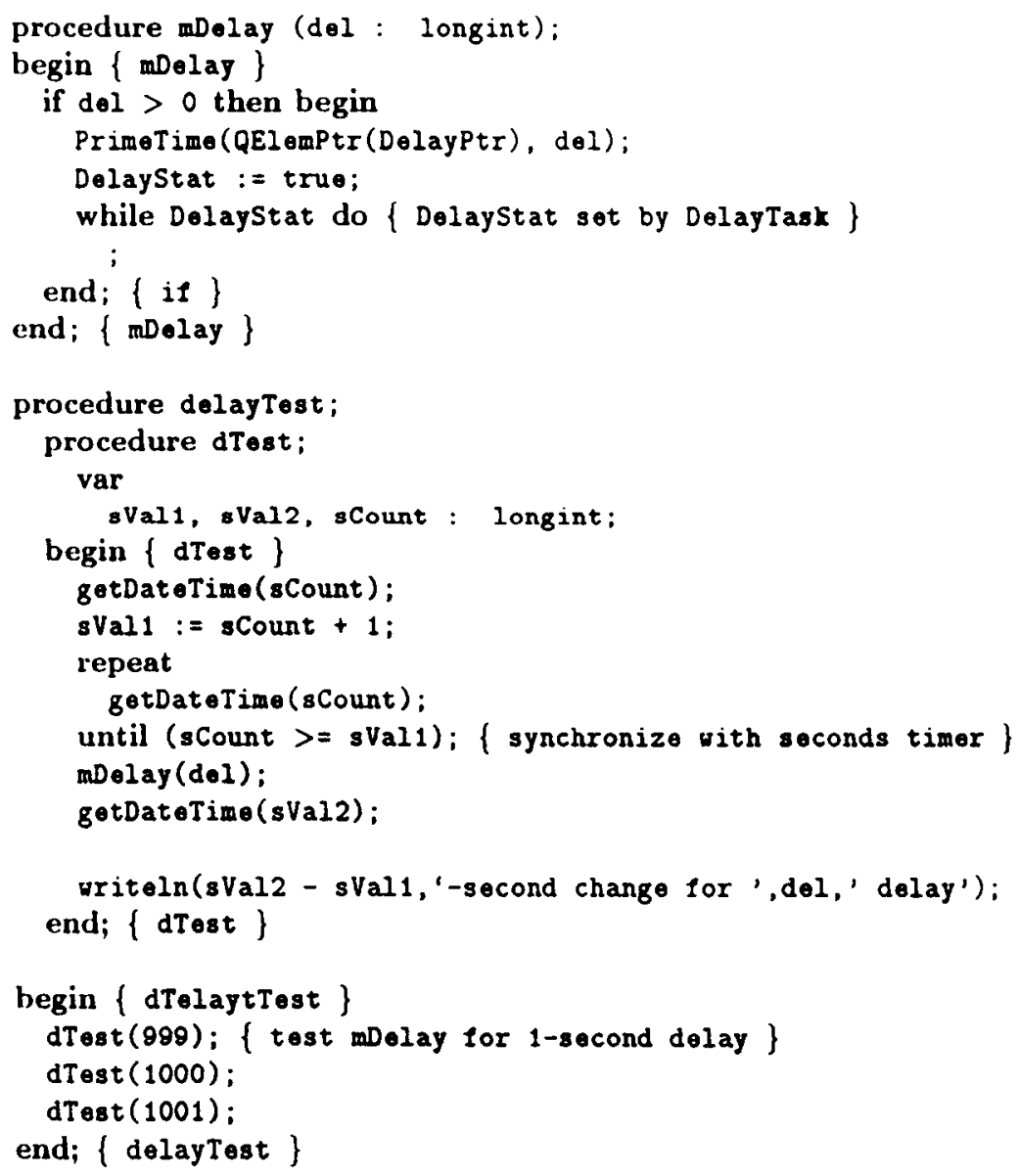

Figure 6. Procedure for millisecond delay. 
I-145 of Inside Macintosh (Apple Computer, 1985), or pages 99-120 of Macintosh Revealed (Chernicoff, 1985).

\section{Basics}

Although there exist specific Toolbox procedures for the machine-independent display of images, these generally require several ticks for the transfer of an image to the screen. One way to overcome this problem is to draw the image directly on the screen via Toolbox commands. This will work for extremely simple images, but it will still be susceptible to problems of screen synchroni- zation if flicker-free display is required. The more general approach described here is to store images prior to display, either by using graphics commands on an off-screen port or by directly loading preassembled images from some other source. These are then copied directly to the screen buffer for display. If data can be transferred quickly enough, an image of arbitrary complexity can be placed on the screen within one refresh cycle.

Procedure setUpBuffers in Figure 7 shows how image buffers (together with their associated graphics ports) can be set up. The subroutine getParams first sets the values

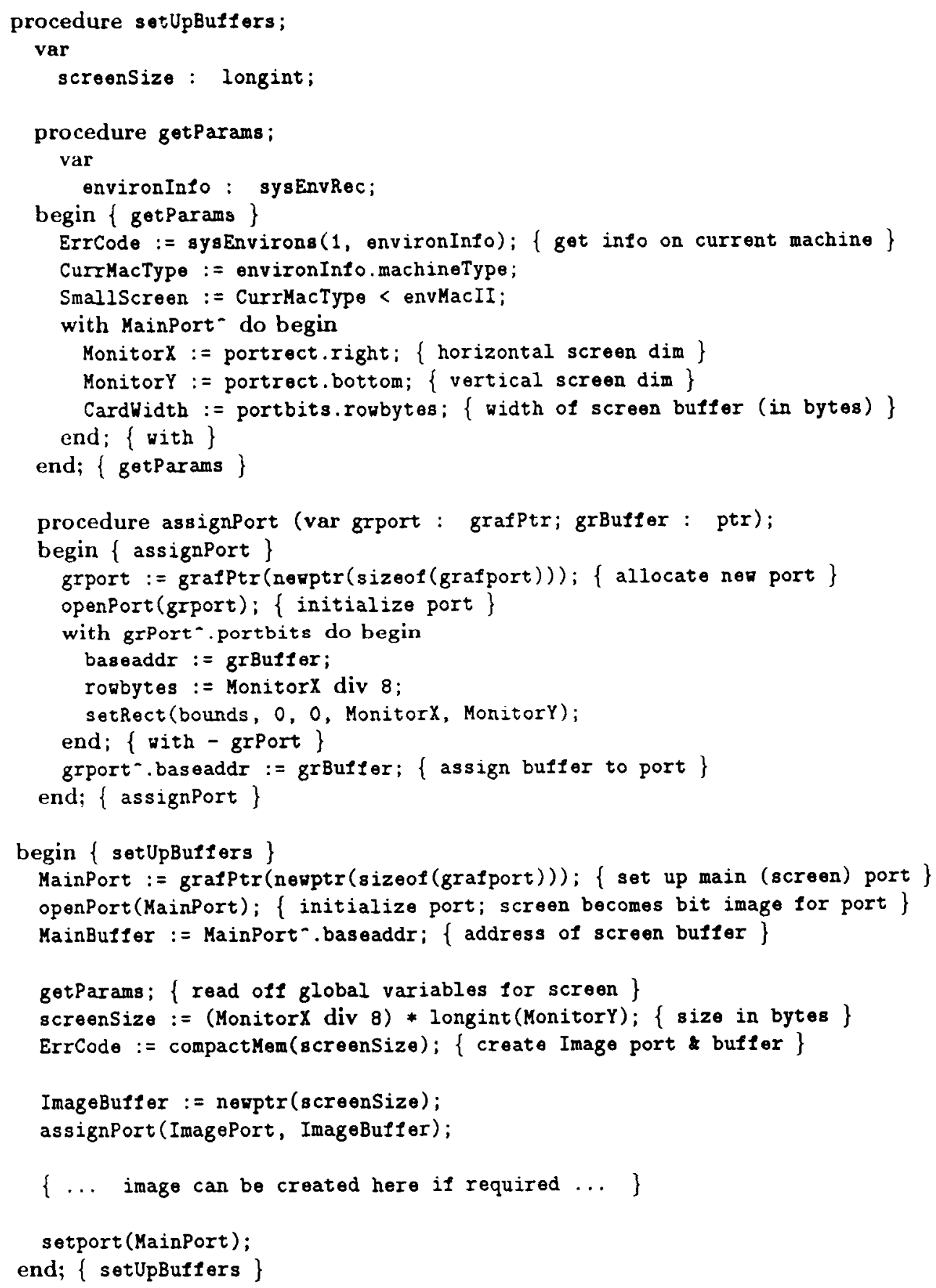

Figure 7. Procedure for setting up image buffers. 


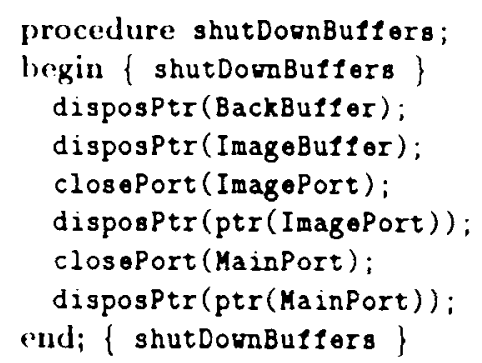

Figure 8. Procedure for shutting down image buffers.

of all global variables pertaining to the buffers, such as the horizontal and vertical dimensions. Memory is allocated for the corresponding bit images, and the associated graphics ports are then set up. The complementary procedure shutDownBuffers in Figure 8 shows how the memory may be released after use.

Owing to differences in video circuitry, it is useful to distinguish between two groups of machines: small-screen Macs and large-screen Macs. The former group encompasses the $128 \mathrm{~K}, 512 \mathrm{~K}$, Mac Plus, and standard SE models. These have in common (1) a fixed (standard) screen 342 pixels high and 512 pixels wide, (2) a screen refresh synchronized with the tick timer, running at $60.15 \mathrm{~Hz}$, and (3) an alternate video buffer that can be displayed by clearing bit 6 in VIA data register $A$.

Large-screen machines, which include the Mac II and Mac IIx, operate quite differently. These machines have (1) video cards containing screen buffers designed for a variety of screen sizes, (2) a screen refresh that depends on the particular monitor used, and (3) no alternate screen buffer. Although many different types of monitor are possible, one of the most commonly used is the standard Mac II monitor. This has a screen 480 pixels high and 640 pixels wide, with a refresh rate of $67 \mathrm{~Hz}$. The procedures described here will be designed for this monitor. It is a simple matter, however, to adapt them to monitors of other sizes.

It should be noted that because of the two different kinds of video circuitry, the procedures described below depend on the kind of machine running the program. However, the global variable SmallScreen (determined in procedure getParams in Figure 7) can be used to select the appropriate procedures for the machine being used, as is shown in the simple routıne screenTest in Figure 9. In this way, routines can achieve a fair degree of machine-independent operation.

\section{Small-Screen Macs}

The (main) screen buffer for small-screen Macs has a size of 342 rows $\times 512$ columns; images to be displayed must therefore be stored in bit images of size 21888 bytes. The images themselves are created prior to display, either by carrying out a set of standard graphics commands using the port associated with the image (cf. setUpBuffers in Figure 7), or by directly loading the images in from some other source. The number of images that can be set up in this fashion is limited only by the available RAM.

A routıne for displaying images within one screen refresh is given by the procedure movelmage in Figure 10 . This routine relies on a sequence of blockMove commands that transfer data between the buffer and the screen within 18-24 msec, depending on the particular machine used. Although data transfer using this method is not quite fast enough to move an image within one screen refresh, careful synchronization with the beginning of a video scan (by the use of mDelay) allows the location of the pixels being loaded into RAM to lag behind the scanning beam during the first tick, and to lead it during the second tick. Consequently, the image is displayed on the screen within one refresh cycle. Thus, provided that a predisplay duration of one tick is reserved for each change of display, an indefinite number of images can be transferred to the screen without flicker. It is important to realize that once an image has been moved to the screen, it must remain there during the predisplay interval of the succeeding image. Thus, images must remain on the screen for a minimum of two ticks. Apart from this, however, there are no constraints on the duration of a screen display.

An important note concerning moveImage is that it uses a sequence of blockMove commands, rather than just a

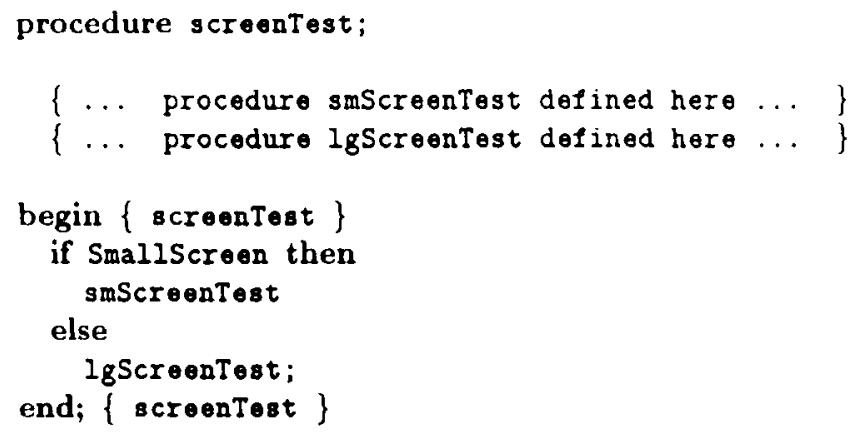

Figure 9. Machine-independent procedure to test fast image transfer. 
single instruction. This is necessary because blockMove transfers data in the reverse direction from that of the scanning beam, beginning at the end of the buffer and ending at the start. If a single blockMove instruction is used to transfer an entire image, this will completely disrupt the synchronization of data transfer with the scanning beam, resulting in a noticeable distortion of briefly displayed images.

\section{Large-Screen Macs}

The wide variety of monitor configurations possible for large-screen machines introduces a few more complications into video display. To begin with, large-screen machines use slots to handle monitor operations, each slot being assigned to a particular monitor. Furthermore, monitors may be driven by different video cards, each with its own particular characteristics. These matters are dis-

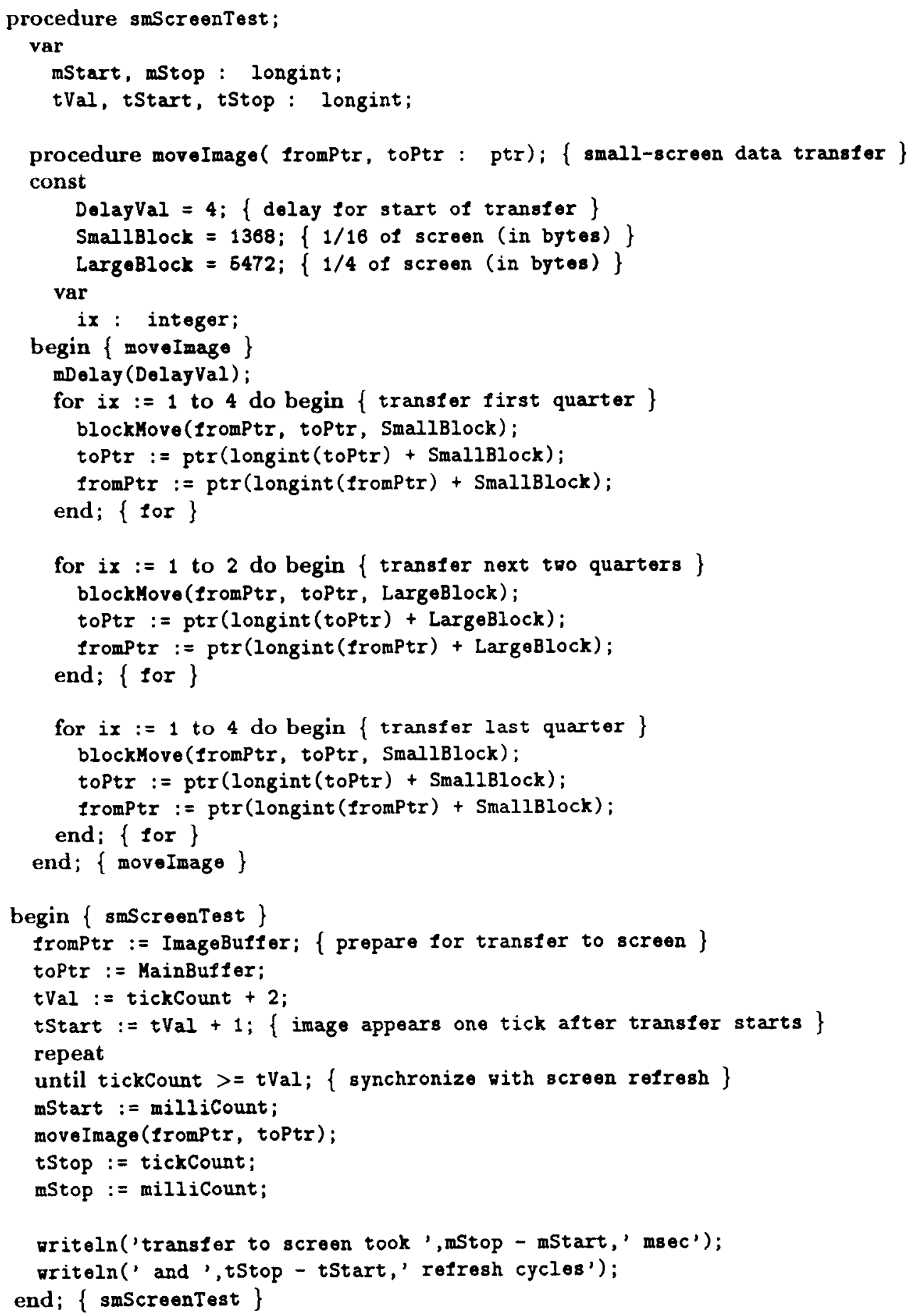

Figure 10. Procedure for fast image transfer on the small-screen Mac. 
cussed on pages V-426-V-428 and V-566-V-569 of Inside Macintosh (Apple Computer, 1988), so they will not be described in detail here. In the interests of simplicity, attention will be restricted to single-slot systems. It is assumed that the slot number (which must be between 9 and 14 ) is known.

The basic ideas behind fast display on large-screen Macs are very similar to those for small-screen systems. Since
tickCount cannot be used to synchronize data transfer with screen scans, it is necessary to use a vertical retrace task for this purpose. In a fashion analogous to that used in the Time Manager queue, the vblCount field of a vertical retrace task is decremented at the beginning of each screen refresh cycle; when this value reaches zero, the associated procedure is executed (see pp. V-566-V-569 of Inside Macintosh, Apple Computer, 1988, for further details).

$\{A+\}$ \{asynchronous \} procedure Videoproc; \{ increment video counter \} begin

videocount $:=$ videoCount $+1 ;$ \{increment global counter \}

videoTask. vblCount $:=1$; \{ reset .vblCount field \}

end;

$\{A-\}$

$\{A+\}\{$ asynchronous $\}$ procedure slotproc;

begin

Errcode := dovBlTask(Currslot); \{carry out vertical-rotrace task end;

$\{A-\}$

procedure sotUpVCounter;

begin \{ setUpvCounter \}

Cursslot : = NoSlot; d dummy value

if not Smaliscreon then begin \{ set up active slot\}

videocount $:=0 ;\{$ initialize global value

Videoptr $:=$ ovideoTask;

with Videoptr- do begin \{ set up vertical-retrace task \}

qType := Ord (vType);

vbladdr := OVideoproc;

vblCount $:=1$;

vblPhase $:=0$;

end; $\{$ vith $\}$

SlotPtr: : OSlotTask;

with SlotPtr do begin (set up slot-interrupt task\}

sqType $:=6 ;\{$ slot queue type

sqprio : $=255$; (highest priority \}

sqAddr $:=$ OSlotProc;

sqparm : $=0$;

end; $\{$ uith $\}$

readin(CurrSlot); \{ must have value between 9 and 14 \}

ErrCode := slotVInstall (QElemPtr(VideoPtr), CurrSlot);

ErrCodo := SIntInstall (SlotPtr, Currslot);

end; $\{$ if $\}$

end; \{ setUpvcounter \}

procedure shutDownVCounter;

begin

if Currslot $<$ NoSlot then begin

ErrCode := SIntRemove (SlotPtr, CurrSlot);

ErrCode := slotVRemove(QElemPtr(VideoPtr), CurrSlot); end;

end ;

Figure 11. Procedures for setting up and shutting down video counter. 
The procedure setUpVCounter (Figure 11) shows how the vertical retrace task VideoTask is set up. The VideoProc procedure associated with this task increments the global counter videoCount each time it is called. It also resets the vblCount field so that VideoTask is called during each vertical blanking interval.

Once it has been set up, VideoTask is installed into the vertical retrace queue associated with the slot. The slotinterrupt task SlotTask is then placed into the slot-interrupt queue. This task, set up just after VideoTask, is called at each slot interrupt, using doVBLTask to carry out the tasks in the queue associated with the slot. Since an interrupt is generated at the beginning of every vertical blanking interval, this ensures that VideoTask increments the value of videoCount, so that it functions analogously to tickCount.

Exactly as in the case of the Time Manager queue, care must be taken to shut down the vertical retrace tasks before exiting the program. Procedure shutDownVCounter in Figure 11 shows how this can be done.

The screen buffer for a large-screen Mac is located in the RAM of the video card being used for display. The width of the screen buffer (in bytes) can be read off the rowbytes field of the screen bitmap, as shown in getParams in Figure 7. When a monitor of width $w$ is used, the displayed image is held in the $w$ leftmost columns of the buffer, the other columns remaining unused. Buffers for bit images, of course, need only be large enough to hold the images to be displayed.

The procedure lgScreenTest (Figure 12) shows how a bit image can be transferred to a standard monitor of size $480 \times 640$ pixels. Data transfer is based on the use of typecasting, which allows rows of the image to be declared as arrays. Assigning these arrays to the corresponding locations in the screen buffer means that only the image bits are replaced, rather than the entire contents of the buffer. In this way, large-screen machines can generally move an image to the screen within one refresh cycle. For example, using the procedure lgScreenTest on a standard Mac II, it has been found that an image can be transferred to the screen in $14 \mathrm{msec}$, which is less than the $15 \mathrm{msec}$ used for a screen refresh. Thus, an indefinite number of bit images can be sequentially displayed on this screen at refresh rates.

Although this technique is rather specialized, it can be adapted for use on other machines and other screen sizes simply by typecasting rows into arrays of the appropriate size. If the screen size is so large that an image cannot be transferred within one refresh, the synchronization technique for small-screen Macs can be applied. If the image still cannot be placed on the screen within a single refresh, transfer can be broken down into several

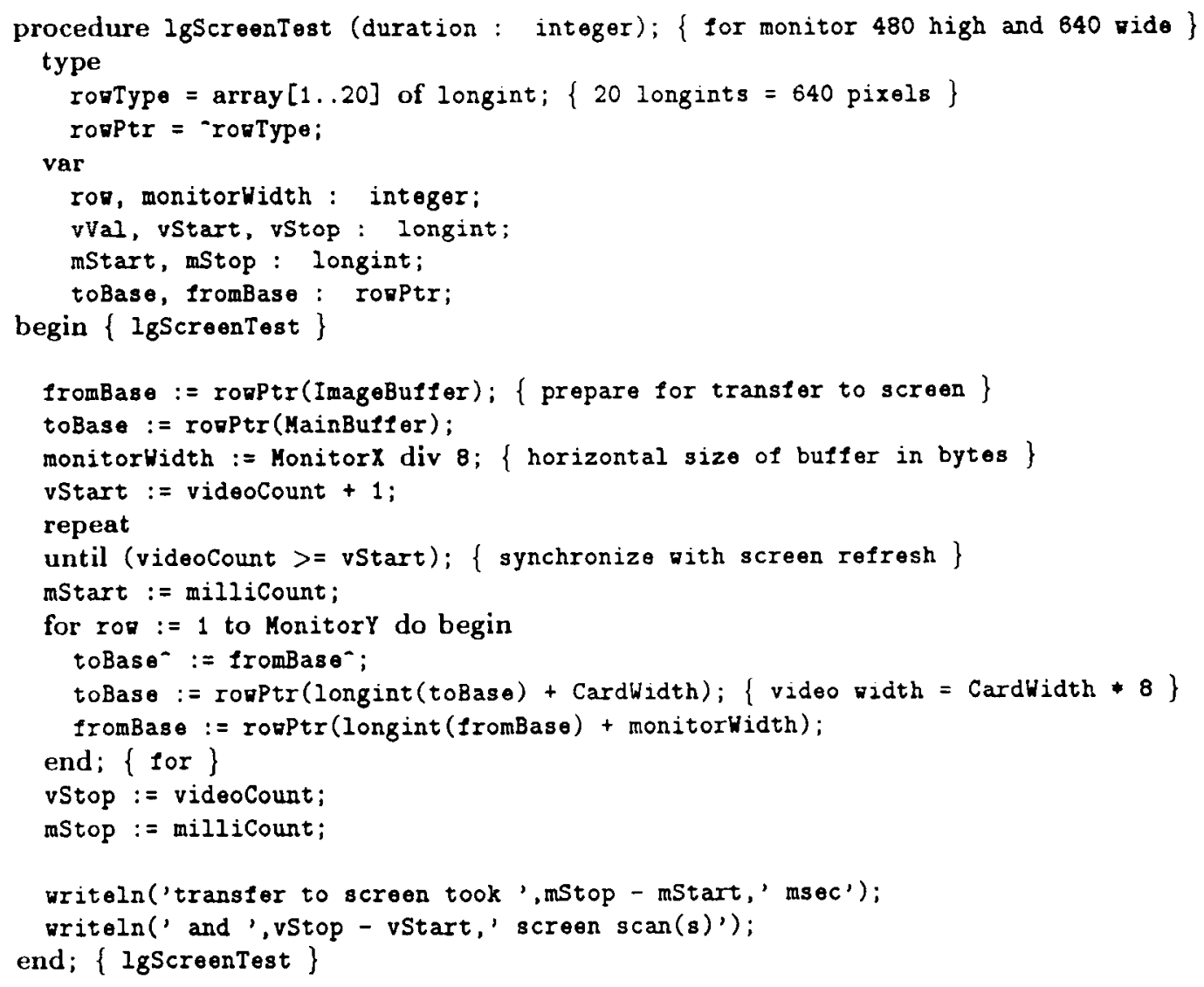


phases, each of which transfers a number of evenly-spaced rows. This kind of interlacing technique can easily be programmed with the techniques described here, and it will ensure that the image can always be placed on the screen without any disruption.

Although it has not been done here, it is easy to use the above routines to create a general display system that can be used for any monitor. It is only necessary to create a set of typecasting operations, and then to use the appropriate one for transfer. As such, this routine would be a variant of the movelmage routine given in Figure 10.

\section{Combining Timing and Display}

The procedures described above can be combined and/or modified to perform various tasks. For example, it is possible to combine routines from anchorTest and screenTest into a simple routine that measures the time from image presentation to keypress. Note that the smallscreen version must ensure that the start anchor is one tick greater than the actual value of tickCount at the beginning of data transfer, since the image does not begin to appear on the screen until the transfer is halfway completed.

This procedure suffers from one minor restrictionnamely, if a keypress occurs while data is being moved, the response will not be noticed until after the data transfer has ended. This might be a problem for processes such as animated display, where data transfer takes place during a significant fraction of the time. However, transfer can be carried out in blocks, each of which requires less than a millisecond to be moved. Precise timing can then be carried out, checking the status of OSEventAvall after each of these moves.

Finally, it should also be pointed out that the display routines can easily be modified to allow the rows of an image to be transferred to the screen at any rate and in any order. Thus, for example, if interlacing of the displayed rows is done carefully, an image can be transferred over an extended period of time, leading to a smooth transition from the previous image on the screen.

\section{REFERENCES}

App1F Computer (1985) Insude Macinush. Volumes I-III Menlo Park. CA Addison-Wesley

Apple Computer (1986) Inside Macintosh Volume IV Menlo Park. CA Addison-Wesley

Apple Computer (1988) Inside Macintosh Volume V Menlo Park. CA Addison-Wesley

APPIE COMPUTER (1988) Macinsosh famsly hardutare reference Menlo Park, CA Addison-Wesley

CHERNICOFF, S (1985) Macintosh revealed Volume I Unlocking the Toolbox Hasbrouck Heights. NJ Hayden

Kiel.er. J M. Hicisins, T S (1988. November) Precision tuming options for the Apple Macintosh family of computers Paper presented at the Eighteenth Annual Meetung of the Society for Computers in Psychology. Chicago

Lane, D M.. Ashby, B (1987) Psychlib A library of machine language routınes for controlling psychology experiments on the Apple Macintosh computer Behavior Research Methods, Instruments, \& Computers, 19, 246-248

REED COLLEGE (1985) Rascal user manual Macintosh language for real time $/ / O$ oriented development Portland, OR Metaresearch.

Westall, R , Perkey, M N., Chute, D L. (1986) Accurate millisecond tıming on Apple's Macintosh using Drexel's MilliTimer Behavior Research Methods. Instruments, \& Computers, 18, 307-311 